\title{
Konflikte in der Beratung: Individuelle, interpersonale und organisationale Perspektiven
}

\author{
Silja Kotte
}

Online publiziert: 14 . Oktober 2021

(C) Springer Fachmedien Wiesbaden GmbH, ein Teil von Springer Nature 2021

Das Wort Konflikt leitet sich vom Lateinischen confligere ab, was „,zusammentreffen, kämpfen“ bedeutet. Bei einem Konflikt treffen also widerstrebende Motive, Interessen, Zielsetzungen oder Wertvorstellungen aufeinander. In der Regel verbindet sich mit dem Begriff Konflikt die Annahme, dass verschiedene Akteure (Personen, Gruppen oder Organisationen) aufeinandertreffen, es sich also um interpersonale, Gruppen- oder Organisationskonflikte handelt. Konflikte können jedoch auch intrapersonal sein, also innerhalb einer Person stattfinden, wenn diese widerstrebende Motive oder Regungen in sich verspürt, etwa in Form von Entscheidungs- oder Rollenkonflikten.

Konflikte in all diesen unterschiedlichen Formen können in der Beratung relevant sein. Konflikte und deren „Management“ zählen in Coachingmarktumfragen zu den Top 5 der zentralen Themen im Coaching (vgl. Rauen 2020; Middendorf 2020) neben Themen wie dem Hereinfinden in neue Aufgaben/Positionen, der Reflexion der eigenen Person und Rolle sowie der Persönlichkeits- und Potenzialentwicklung. Berater/innen werden als Expert/innen bei der Unterstützung im Umgang mit Konflikten angefragt. Berater/innen können im Kontext von Beratungsaufträgen selbst in die Konfliktdynamik im Klient/innensystem „,hineingezogen“ werden (Louis und Fatien Diochon 2014). Und Berater/innen sind gefragt, sich einen Überblick über die Konfliktdynamik und -konstellation zu verschaffen und Entscheidungen darüber zu treffen, welche Stakeholder in die Konfliktbearbeitung wie eingebunden werden sollen, d.h. mit wem in welchem Setting und mit welchem Verfahren der Konfliktbearbeitung (z.B. Coaching, Supervision, Mediation, Organisationsberatung ...) der Konflikt angegangen werden soll.

Prof. Dr. Silja Kotte $(\bowtie)$

Fachbereich Psychologie, HMKW Hochschule für Medien, Kommunikation und Wirtschaft,

Solmsstraße 18, 60486 Frankfurt, Deutschland

E-Mail: s.kotte@hmkw.de 
Vor diesem Hintergrund geht der Themenschwerpunkt Konflikte in der Beratung Konflikten und ihrer Bearbeitung im Rahmen von arbeitsweltlicher Beratung auf unterschiedlichen Ebenen nach: vom Umgang mit intrapersonalen, innerpsychischen Konflikten von Coaching-Klient/innen bis hin zu einer Analyse von Konfliktdynamiken und deren Bearbeitung auf organisationaler Ebene. Dabei kommen auch Überlegungen zur Indikation und Abgrenzung verschiedener Beratungsformate für die Konfliktbearbeitung in Organisationen zum Tragen. Zusammen sollen die fünf Beiträge des Themenschwerpunkts zum Nachdenken über das eigene Verständnis von Konflikten, die mit ihnen verbundenen Chancen und Risiken und Möglichkeiten ihrer Bearbeitung in der Beratung anregen.

Der Beitrag von Wilfried Kerntke fokussiert auf die organisationale Ebene. Unter dem Titel „Unternehmen im Konflikt“ geht er auf die spezifische Dynamik von Konflikten in Organisationen ein. Er erläutert, welchen Bezug die Konfliktdynamik zur Entwicklung der Organisation entlang der Abfolge von Pionier-, Differenzierungs-, Integrations- und Assoziationsphase aufweist, führt die Unterscheidung zwischen Protagonisten, Zaungästen und Stakeholdern des Konflikts ein und verdeutlicht, inwiefern sich bei den Stakeholdern des Konflikts - zumindest vorübergehend - eine „organisationale Vernunft“" zeigt, die sich in der Konfliktbearbeitung nutzbar machen lässt. Anschaulich erläutert er sowohl die organisationale Konfliktdynamik als auch Vorgehensweisen zur Konfliktbearbeitung anhand eines Praxisbeispiels.

Die folgenden beiden Beiträge beschäftigen sich mit intrapsychischen Konflikten von Coaching-Klient/innen, und zwar auf der Grundlage der Operationalisierten Psychodynamischen Diagnostik (Arbeitskreis OPD 2006). Die OPD versteht intrapsychische Konflikte als ,unbewusste innerseelische Zusammenstöße entgegengerichteter Motivbündel“" (ebd., S. 96), die das individuelle Erleben und Verhalten stark prägen. Die OPD nimmt an, dass bei verschiedenen Personen unterschiedliche intrapsychische Konflikte im Vordergrund stehen.

Michael Stoll fokussiert in seinem Beitrag auf den intrapsychischen Konflikt Dominanz vs. Unterordnung. Er geht der Frage nach, wie Coaching Coachees bei einer situationsangemessenen Selbstregulation im Hinblick auf dieses Spannungsfeld unterstützen kann. Basierend auf einer qualitativen Interviewstudie arbeitet er die aus Sicht erfahrener Coaches hilfreichen Wirkfaktoren und Interventionen heraus. Dabei finden sich sowohl Faktoren, die für dominanzorientierte und zu Unterordnung neigende Coachees gleichermaßen hilfreich scheinen (z. B. die Reflexion des Interaktionsgeschehens zwischen Coach und Coachee im Hinblick auf Dominanz und Unterordnung), als auch Hinweise auf unterschiedliche Vorgehensweisen im Coaching, je nachdem, zu welchem Pol Coachees eher neigen (z. B. Ressourcenorientierung bei zu Unterordnung neigenden Coachees, wertschätzende Konfrontationen bei dominanzorientierten Coachees).

Claudia Halberstadt nimmt den intrapsychischen Konflikt Abhängigkeit vs. Individuation im Kontext von Einzelcoaching in den Blick, und zwar auf Grundlage einer Interviewstudie mit erfahrenen Coaches, die sie mittels qualitativer Inhaltsanalyse ausgewertet hat. Sie arbeitet subjektive Theorien und Ansätze von Coaches zum Umgang mit dem Abhängigkeit-vs.-Individuation-Konflikt ihrer Coachees im Coaching heraus und veranschaulicht diese mit Interviewzitaten. Dabei zeigt sich die besondere Bedeutung der Gestaltung von Nähe und Distanz in der Arbeitsbezie- 
hung zwischen Coach und Coachee (kontrastierend-komplementär vs. responsiv). Auch die Reflexion der Passung von Person und Rolle/Funktion sowie die Arbeit an individuellen Entwicklungsfeldern (z. B. Emotionsregulation, Kontaktgestaltung) erweist sich als relevant.

Kirsten Schroeter beschäftigt sich mit den Spezifika der Mediation in Organisationen im Spektrum der Konfliktbearbeitungsverfahren. Sie gibt einen Überblick über den aktuellen Stand der Mediation in Deutschland, insbesondere Mediation in Organisationen, und skizziert auch den rechtlichen Rahmen für Mediation. Sie erläutert aktuelle Debatten aus der konkreten Praxis der Mediation insbesondere im Hinblick auf Verbindungslinien und Abgrenzungsmomente von Mediation zu den Formaten Supervision und Coaching und illustriert diese mit Fallvignetten. Abschließend beleuchtet sie genauer das Verhältnis von Mediation und Supervision und schließt mit einem Appell, konfliktbezogen voneinander zu lernen.

Beate West-Leuer geht in ihrem Praxisbericht auf einen speziellen Bereich betrieblicher Konflikte und Konfliktmanagement ein, nämlich das Feld der Arbeitsund Betriebsmedizin. Sie erläutert, dass Arbeits- und Betriebsmediziner/innen zwar keine Konfliktmanager/innen, -moderator/innen oder -mediator/innen sind, aber dennoch intensiv mit betrieblichen Konflikten zu tun haben, mal als Beobachter/innen, mal als Beteiligte. Der Beitrag gibt Anregungen für Konfliktdiagnosen und daraus abgeleitete Interventionsstrategien, die auch eine psychodynamische Tiefenebene miteinbeziehen. Dazu verweist sie auf Konzepte wie Primäraufgabe und Primärrisiko oder die Unterscheidung zwischen intrapsychischen und interpersonellen Konflikten und illustriert diese anhand von Fallvignetten, bei denen Arbeits- und Betriebsmediziner/innen beteiligt sind.

Neben diesen Beiträgen zum Themenschwerpunkt finden sich drei weitere Hauptbeiträge in diesem Heft:

Thomas Bachmann beschäftigt sich mit psychischen Verletzungen, die durch organisatorische Veränderungen entstehen, und betrachtet diese unter einem GestaltCoaching-Ansatz. Er zeigt auf, dass das Kränkungserleben durch organisationale Veränderungen sich oft substantiell auf das Selbstvertrauen von Organisationsmitgliedern auswirkt und dass die Arbeit mit gekränkten Führungskräften im Coaching anspruchsvoll ist. Vor diesem Hintergrund schlägt der Autor einen Gestalt-Ansatz für die Arbeit mit gekränkten Coaching-Klient/innen vor, der sich am Kontaktzyklus der Gestalttherapie orientiert.

Larry Hirschhorn, Thomas Giernalczyk, Martin Holle, Mathias Lohmer und Markus Zimmermann gehen unter dem Titel „Drei kulturelle und organisatorische Welten" darauf ein, wie die Covid-19-Pandemie virtuelle Formen der weltweiten Zusammenarbeit beschleunigt und wie dadurch die Kultur, die Struktur und die Prozesse von Organisationen beeinflusst werden. Sie unterscheiden drei Welten: die bürokratische Welt (B-Welt) mit ihrer Orientierung an Hierarchie, Kontrolle und Vorhersagbarkeit, die Projektwelt (P-Welt) mit den Werten Team, Kooperation und Entwicklung und die mit Freiheit, Autonomie und Unsicherheit verbundene Transaktionsoder Gig-Welt (T-Welt). Sie postulieren, dass jede Welt mit einer bestimmten Kultur einhergeht und so innerhalb von Unternehmen eine multikulturelle Landschaft entsteht, die spezielle Herausforderungen mit sich bringt, u.a. die Frage nach dem 
richtigen Zuschnitt der drei Welten innerhalb des Unternehmens (wieviel braucht es von welcher Welt?).

Der Beitrag von Petia Genkova und Henrik Schreiber beschäftigt sich mit dem Thema Diversity. Insbesondere geht es vor dem Hintergrund des Fachkräftemangels um die Frage, inwiefern Führungskräfte im MINT-Bereich sich der Chancen und Risiken kultureller Vielfalt und der spezifischen Herausforderungen für Menschen mit Migrationshintergrund bewusst sind. Die Ergebnisse einer qualitativen Interviewstudie mit Führungskräften verschiedener deutscher Unternehmen deuten insgesamt auf ein eher geringes Diversity-Bewusstsein bei den befragten Führungskräften hin. Gleichzeitig zeigt sich, dass vergangene und aktuelle Erfahrungen (z.B. internationale Lebenspartner/innen, viel Zusammenarbeit mit internationalen Kolleg/innen, Diversity-Management-Maßnahmen des Arbeitgebers) sich deutlich auf die Einstellungen gegenüber Diversity und auf die Differenziertheit im Hinblick auf das Thema Diversity auswirken.

Der Diskurs von Uwe Böning schließt an den Diskurs aus Heft 3/2021 zum Thema „Neuorientierung für das Business-Coaching: Anfragen zur gesellschaftlichen Verantwortung von Coaches“" an. Der Autor stellt die Frage, ob Coaching eine ikonografische Figur der gegenwärtigen Werteausrichtung unserer Gesellschaft ist. Er beschreibt gesellschaftlich-politische Werte-Entwicklungen der letzten 40-50 Jahre im Westen, speziell in Deutschland, und postuliert, dass Coaching, insbesondere Business-Coaching, die Befindlichkeit unserer Gesellschaft widerspiegelt und dass sich dadurch der hohe Zulauf, den Coaching erfährt, erklären lässt.

Die Kulturanalyse nimmt den Film „Three Billboards Outside Ebbing Missouri“ in den Blick. Dirk Blothner beschreibt diesen als einen Film mit starkem zeitaktuellem Subtext, insofern als dass er einen sehr greifbaren Zugang zu Zuständen von Wut, die er „Wut-Besessenheiten“ nennt, ermöglicht. Der Beitrag zeigt am Beispiel des Films auf, wie solche „Wut-Besessenheiten“ entstehen, wie sie das Zusammenleben von Menschen polarisieren, aber auch wie sie sich in gegenseitige Sorge und damit gemäßigtere Umgangsformen umwandeln können. Der Autor verdeutlicht die Aktualität des Themas, indem er Parallelen zu „Wut-Besessenheiten“ zieht, wie sie gegenwärtig etwa in Demonstrationsparolen oder Hass-Posts im Internet aufscheinen.

\section{Literatur}

Arbeitskreis, O.P.D. (2006). Operationalisierte Psychodynamische Diagnostik OPD-2. Das Manual für Diagnostik und Therapieplanung. Bern: Huber.

Louis, D., \& Diochon, F.P. (2014). Educating coaches to power dynamics: Managing multiple agendas within the triangular relationship. Journal of Psychological Issues in Organizational Culture, 5(2), 31-47.

Middendorf, J. (2020). Coaching-Umfrage Deutschland 2019/2020. Frechen: Büro für Coaching und Organisationsberatung.

Rauen, C. (2020). RAUEN Coaching-Marktanalyse 2020. https://www.rauen.de/cma/. Zugegriffen: 1. Okt. 2021. 